\title{
Conceptions des futurs maîtres du primaire relativement à l'utlisation de la resolution de problèmes en mathématiques
}

\section{LUCIE DEBLOIS \& NANCY VÉZINA}

Université Laval

\section{RÉSUMÉ}

Cet article rapporte les résultats d'une étude portant sur les conceptions de l'enseignement des mathématiques chez des futurs maîtres qui suivent leur premier cours en didactique des mathématiques. Quatre activités d'enseignement, portant sur la multiplication et la division, ont été proposées. Les futurs maîtres ont identifié les forces et les faiblesses lors d'une première discussion en sous-groupes. Ils ont ensuite été invités à valider et justifier leurs choix en groupe d'experts, selon la modalité proposée en apprentissage coopératif. Enfin, de retour dans leur groupe initial, ils ont identifiés ce qu'ils considèrent important à retenir pour l'apprentissage et l'enseignement de la notion mathématique en jeu. Une analyse des 88 questionnaires, complétés durant les discussions, a été réalisée. Nos résultats laissent apparaître certaines conceptions à l'égard de ce type d'activité d'enseignement notamment l'importance pour eux d'illustrer un problème par un matériel et d'être conforme aux objectifs d'apprentissage. En outre, le questionnement est vu comme un générateur de confusions chez les élèves. Les discussions en groupe d'experts semblent susciter un approfondissement de ces conceptions dans le cas où les activités d'enseignement ne posent pas de problème de contenu mathématique 
aux futurs maîtres. Les résultats obtenus permettent de dégager des moments de tension qui guideront nos interventions dans la classe de didactique à l'université.

\section{ABSTRACT}

This article discusses the results of a study on preservice teachers's conceptions of a teaching activities included problem-solving. Four teaching activities on the concept of multiplication and division were porposed during a course. First, the preservice teachers were asked to identifiy the strengths and weaknesses. They were then invited to validate and justify their choice with expert subgroups according to cooperative learning strategies. Finally, they determined what should be retained for the learning and teaching of these mathematical notions. Analysis of the 88 questionnaires allowed us to study the conceptions of teaching activities used the problem-solving as a teaching strategie. It appears conceptions about these type of teaching activitics. For example, preservice teachers viewed the illustration of a problem as the primary strength, but asking questions as a web to create confusion in pupils. When the mathematics content present no confusion, they can reflect more on the activities of pupils. The results help us to identify tension who will guide pedagogic intervention for the program.

\section{PROBLÉMATIQUE}

Les études en didactique des mathématiques ont tenté d'identifier divers facteurs ayant une influence sur les pratiques privilégiées dans la classe de mathématiques. Certains travaux se sont intéressés aux enseignants et aux enseignantes en exercice alors que d'autres ont été réalisés auprès de futurs maîtres. L'étude de Wideen, Mayer-Smith et Moon (1998) permet de reconnaître l'étendue et la diversité des recherches qui tentent de cerner le développement de l'apprentissage de l'enseignement. Alors que certaines études s'attardent aux conceptions des futurs maîtres, d'autres s'intéressent à l'influence des programmes de formation ou encore au phénomène d'insertion professionnelle durant la première année d'enseignement. Nous situons cette étude à l'intérieur de 
celles qui s'intéressent aux conceptions des futurs maîtres sous l'influence d'un dispositif d'enseignement dans une classe universitaire.

Noël et Mura (1999), Jaworksi (1994), Ernest (1989, 1991), Underhill (1988) et Dionne (1988) entre autres, se sont attardés à l'identification des conceptions des mathématiques comme facteur ayant une influence en classe de mathématiques.' Civil (1993), Johnston (1990) et Ross (1987) ont étudié certaines sources génératrices de ces conceptions. Ils ont reconnu l'importance des premières expériences d'apprentissage, par exemple au primaire, ou encore l'expérience des cours de mathématiques centrés sur la résolution de problèmes (Emenaker 1995). Toutefois, pour Britzman (1991) les conceptions joueraient le rôle de filtre, mais de façon plus complexe qu'on ne pourrait le croire. En effet, les conceptions susciteraient la création de mythes comme celui-ci: l'enseignant qui n'établit pas le contrôle ne pourra faire apprendre ses élèves.

Un deuxième facteur ayant une influence en classe de mathématiques touche la formation reçue sur le développement de la pensée des élèves (Fenema, Carpenter, Franke, Levi, Jacob \& Empson, 1996). Alors que cette formation semble permettre aux enseignants en exercice de prendre plus de temps pour écouter les élèves et pour les amener à trouver plusieurs solutions au moment de résoudre des problèmes, une formation semblable ne semble pas donner les mêmes résultats chez les futurs enseignants. En effet, Nesbitt Vacc et Bright (1999) ont observé que les futurs maîtres développent une connaissance de l'enseignement qu'ils qualifient de fragile. Ainsi, les futurs maîtres modifieraient leurs conceptions pour une orientation plus constructiviste et enseigneraient selon les principes proposés par la formation Cognitively Guided Instruction dans certains contextes seulement. Les auteurs posent posent deux hypothèses pour expliquer cette différence: la première concerne la différence d'expérience d'enseignement, la seconde est relative à la nécessité de développer simultanément une connaissance de la pensée et de l'apprentissage des élèves de même qu'un ensemble de stratégies d'enseignement.

Il nous semble donc souhaitable de lier le contenu disciplinaire à enseigner et le développement de la pensée des élèves au moment de 
former des futurs maîtres à l'enseignement des mathématiques. En effet, compte tenu du fait que la plupart des contenus à l'étude au primaire portent sur des notions que les futurs maitres utilisent depuis longtemps, ces notions semblent simples. Une relation entre le contenu disciplinaire à enseigner et le développement de la pensée de l'élève pourrait leur permettre de reconnaître la complexité de l'apprentissage de notions, en apparence très élémentaires.

Nous inscrivons ainsi cette étude dans la foulée de celles qui analysent les dispositifs de formation mis en place (Bednarz, Gattuso, \& Mary, 1997; Hong, 1995; Westerman, 1991; Wilcox, Lanier, Schram, \& Lappan, 1992). L'ensemble des recherches, portant sur les interventions à court ou long terme qui sont réalisées auprès des futurs enseignants, laissent apparaître divers problèmes. Certains problèmes sont liés à la relation entre les perspectives philosophiques de l'enseignant qui accueille l'étudiant dans sa classe et les perspectives philosophiques impliquées dans le programme d'étude (Nesbitt Vacc \& Bright, 1999). alors que d'autres sont amenés par la différence entre les attentes des formateurs et des futurs enseignants. Wideen, Mayer-Smith, et Moon (1998) posent d'ailleurs l'hypothèse selon laquelle une confrontation entre les conceptions de l'apprentissage des futurs enseignants permettraient d'entrer dans une première étape du processus de l'apprentissage de l'enseignement. Nous nous intéressons donc à l'étude des retombées d'un dispositif d'enseignement, en classe de didactique, dans lequel les conceptions des futurs maîtres du primaire sont activées et discutées en début de formation.

Une étude de Bednarz (2000), menée auprès d'enseignants et d'enseignantes en exercice, a déjà révélé qu'une discussion portant sur un pré test conduisait à s'attarder aux connaissances antérieures, aux stratégies de résolution, aux raisonnements et aux difficultés de leurs élèves. Dans le cas où les discussions portaient sur des activités d'enseignement, ce sont plutôt les habitudes d'enseignement et le contenu à enseigner qui ont fait l'objet de débats. L'étude de Vacc et Bright (1999) nous sensibilise à la différence de résultats qu'il est possible d'obtenir auprès des futurs maîtres compte tenu de l'absence d'expérience d'enseignement. Nous choisissons donc de susciter des 
discussions chez des futurs maîtres enseignants à partir de certaines activités d'enseignement en mathématiques. Quelles conceptions explicitent-ils? C'est la question à laquelle nous nous sommes attardées.

\section{Comment lier contenu disciplinaire à enseigner et développement de la pensée des élèves}

Les activités d'enseignement se réalisent au moment de présenter aux élèves des situations d'apprentissage. En didactique des mathématiques, divers modèles permettent une réflexion au moment de la planification d'une situation d'apprentissage. Par exemple, Vergnaud (1991) utilise la notion de situation pour désigner essentiellement des situationsproblèmes pour lesquelles l'élève peut ou non disposer des connaissances nécessaires à leur résolution, ce qui peut apporter une exploration et une nouvelle réflexion. Brousseau (1986), quant à lui, appelle situation didactique le jeu d'appropriation de connaissances scolaires réalisé par l'élève. Ainsi, l'élève est placé non seulement devant un problème à résoudre, mais dans un milieu. Ainsi, le matériel utilisé, le choix du problème présenté par le maître, le questionnement de ce dernier, de même que les discussions entre les pairs sont autant de composantes de la situation d'apprentissage qui devient un milieu d'apprentissage. Brousseau distingue alors les situations d'action des situations de formulation, de validation ou d'institutionnalisation. Ces modèles, présents dans plusieurs recherches en didactique des mathématiques, semblent complexes pour le futur maître qui débute. Nous nous tournons donc vers une définition proposée pour les maîtres en exercice.

Selon les orientations du ministère de l'Éducation du Québec (MEQ), une situation d'apprentissage se présente en trois phases: la phase de préparation, la phase de réalisation et la phase d'intégration. La phase de préparation permet au maître d'activer les connaissances antérieures des élèves de manière à encourager l'exploration de diverses avenues pour résoudre le problème posé. La lecture du fascicule $\mathrm{L}$ (MEQ, 1988) précise qu'au moment de la phase de réalisation:

l'enseignant guide, propose, questionne. Il aide l'élève à objectiver son action, fait des suggestions, donne l'information jugée trop difficile à découvrir. Il incite l'élève 
à poursuivre ou à reprendre certaines tâches, observe et soutient l'élève qui éprouve des difficultés. Bref, il facilite le traitement du contenu d'apprentissage (p. 47).

Précisons que cette phase présente généralement un défi aux élèves et ce, par la présentation d'un problème. Enfin, la phase d'intégration cherche à susciter l'objectivation et la prise de conscience des apprentissages réalisés. Pour les besoins de cette étude, nous avons donc privilégié la définition du MEQ. En outre, nous nous attardons aux conceptions explicitées par les futurs maîtres lors de la présentation d'une phase de réalisation.

Dans ce cadre, la résolution de problème est vue comme une stratégie d'intervention à privilégier afin de susciter l'élaboration d'un sens à donner aux concepts mathématiques. L'étude d'Emenaker (1995) a permis de reconnaître que des cours de mathématiques, centrés sur la résolution de problèmes, amenaient les futurs maîtres à reconnaître que plus d'une démarche permettait d'arriver à une bonne réponse et que la compréhension était plus importante que la mémorisation de règles. Toutefois, les futurs enseignants sont-ils en mesure de reconnaître l'apport de cette stratégie d'enseignement dans le développement de la compréhension des élèves?

Nous savons que dans la pratique quotidienne des écoles, la notion de résolution de problèmes est multiple. Miller (1996) a d'ailleurs identifié diverses conceptions et leurs implications dans les pratiques des enseignants et des enseignantes. Ainsi, la résolution de problèmes peut être vue comme la recherche de modèles ou de régularités. Elle est vue aussi comme une expérience de recherche et de créativité qui suscite l'exploration et la collaboration entre les élèves, ou encore comme le regroupement de problèmes d'application et d'exercices qui apportent la construction d'un ensemble de "ruses" et d'habiletés. Le programme du ministère de l'Éducation du Québec (1988) propose de concevoir la résolution de problèmes comme un moyen pour initier les élèves aux modes de pensée et d'expression mathématique. La résolution de problèmes devient une stratégie d'intervention qui amène les élèves à développer leur compréhension à travers des situations d'apprentissage qui donnent du sens aux concepts mathématiques. Le rôle de 
l'enseignant et de l'enseignante est alors de créer un environnement qui suscite une réflexion chez l'élève.

L'étude des conceptions des futurs maîtres relativement à une phase de préparation nous a déjà permis de reconnaître l'intérêt que les futurs maîtres accordent au matériel, aux expériences des élèves et à l'importance de limiter les difficultés (DeBlois, Uwimana, \& Vézina, 1998). Toutefois, puisque les futurs maîtres croient qu'il est souhaitable de limiter les difficultés dans une situation donnée, comment comprennent-ils le concept de résolution de problèmes comme modalité d'intervention? Accordent-ils aux élèves le droit d'utiliser différentes procédures pour résoudre un problème? Comment réagissent-ils lorsque nous leur présentons une situation d'apprentissage planifiée selon un ensemble de questions à adapter aux élèves plutôt que selon une explication linéaire de règles? Les études de Benken et Wilson (1996) de même que celle de Steele (1994) ont permis de reconnaître l'importance des exemples spécifiques de la classe, de l'apprentissage coopératif et de la manipulation de concepts comme facteurs d'influence sur les conceptions des futurs maîtres. La situation d'apprentissage offerte à la discussion durant le cours de didactique, invite les futurs maîtres à imaginer les réactions des élèves. Elle est alors considérée comme étant une activité d'enseignement.

\section{Les problèmes ayant une structure multiplicative}

Nous nous attarderons plus particulièrement à la mise en œuvre de problèmes ayant une structure multiplicative. Ces problèmes impliquent, entre autres, la multiplication et la division (Vergnaud, 1991). À l'instar des travaux de Zazkis et Campbell (1994), l'étude des examens déjà réalisés par des futurs maîtres nous sensibilise aux difficultés qu'ils éprouvent lorsqu'il s'agit d'élaborer des situations d'apprentissage portant sur les structures multiplicatives. En effet, les futurs maîtres semblent voir la multiplication essentiellement comme une addition répétée. De plus, ils confondent très souvent le sens mesure et le sens partage qui apparaît dans les problèmes de division.

Nous nous sommes intéressées à connaître, dans un contexte de négociation de connaissances, le fonctionnement implicite que les futurs 
maîtres donnent aux problèmes ayant une structure multiplicative. Compte tenu des difficultés précisées précédemment, nous avons proposé à la discussion des activités d'enseignement dans lesquelles différents problèmes sont présentés. La multiplication fait d'abord intervenir la réunion d'ensembles équipotents. Dans le premier cas, l'activité d'enseignement laisse surgir une des propriétés de la multiplication: la commutativité; dans le deuxième, l'activité d'enseignement laisse surgir une relation entre multiplication et division. Une troisième activité d'enseignement fait ensuite intervenir un produit cartésien. Nos expériences nous montrent que les futurs maîtres voient essentiellement la multiplication comme une addition répétée. Cette activité risque donc de poser des problèmes qui pourraient susciter une discussion intéressante. Enfin, la quatrième activité d'enseignement présente un problème de division faisant appel au sens de partage, celui qui est privilégié par les futurs maîtres, dans lequel une manipulation de l'enseignante expose le sens mesure. Enfin, les futurs maîtres sont informés qu'une des quatre activités d'enseignement pose un contreexemple ou une faiblesse particulière. L'enjeu est donc de repérer cette faiblesse et de justifier leur choix. Ces types de problèmes sont présentés aux élèves du primaire à partir de la troisième année ( 8 ans). L'exploration de ces notions se poursuit jusqu'en sixième année (12 ans).

\section{MÉTHODE}

Selon la classification des recherches réalisées par Boero, Dapueto et Parenti (1996), nous nous situons à l'intérieur d'une méthode où une discussion est suscitée à partir de problèmes professionnels rencontrés en classe. Il s'agit d'étudier l'explicitation des conceptions de l'enseignement de certains concepts mathématiques à travers les difficultés d'une activité d'enseignement. Nous privilégions la formule de l'apprentissage coopératif (Johnson \& Johnson, 1988). Cette modalité d'intervention s'appuie sur certains principes comme l'interdépendance positive, l'apprentissage par une verbalisation significative, le développement d'habiletés coopératives et le développement du sens des responsabilités chez les individus. 
Ces principes ont justifié la mise en place de deux types de groupes. Le premier, appelé le groupe de base (4 personnes), avait pour but de susciter une discussion sur l'ensemble des quatre activités d'enseignement et d'en faire une première analyse pour identifier les forces et les faiblesses. Nous savons que les premières analyses des étudiants et des étudiantes les amènent souvent à porter des jugements (DeBlois \& Squalli, 1997). Nous avons donc privilégié des questions qui laissent une place à cette tendance tout en cherchant à susciter une réflexion plus profonde. Chaque personne de cette première équipe devenait alors responsable d'une des quatre activités d'enseignement et devait faire un résumé de la discussion dans le deuxième groupe. ${ }^{2} \mathrm{Ce}$ deuxième groupe, que nous avons appelé groupe d'experts ( 8 à 10 personnes), avait pour mission de réutiliser les informations des équipes de base en s'attardant à une seule activité d'enseignement. Les futurs maîtres devaient alors déterminer une seule force de même qu'une seule faiblesse et justifier leur choix. Nous avons voulu amener les futurs enseignants à se libérer d'une évaluation dichotomique en approfondissant les raisons de leurs choix. Le retour au groupe de base permettait de poursuivre la discussion en répondant à trois questions plus générales: Que développons-nous chez les élèves? Que retenez-vous pour l'enseignement de cette notion? Que retenez-vous pour l'apprentissage de cette notion?

Ajoutons que les objectifs visés par les activités d'enseignement ont été précisés parce que nous connaissons la difficulté des futurs maîtres à reconnaître les concepts mathématiques dans une situation d'apprentissage. Enfin, le rôle des professeures était, d'une part, d'observer les groupes de travail afin d'évaluer les apprentissages et les interactions. D'autre part, nous cherchions aussi à soutenir les futurs maîtres en encourageant la discussion en groupe et en répondant aux difficultés individuelles.

\section{Populations impliquées dans cette recherche et cueillette des données}

Nous avons voulu réaliser cette étude auprès de futurs maîtres qui sont inscrits pour la première fois à un cours de didactique des mathématiques. Nous avons utilisé cette modalité d'intervention auprès 
de 3 groupes de 75 futurs maîtres. Rappelons qu'ils n'ont encore jamais enseigné. Une fois par semaine, ils réalisent des stages en classe du primaire. Ils en sont donc à leur quatrième rencontre de trois heures sur une série de quinze rencontres.

Dès le premier cours, les futurs maitres se sont familiarisés, avec les différentes composantes d'une situation d'apprentissage (Ministère de l'Éducation du Québec, 1988). Nous avons ensuite consulté, en classe, une des collections utilisées dans les écoles du Québec pour repérer et évaluer les phases de réalisation proposées. Les futurs maîtres constatent alors la différence entre un problème et un exercice. Des lectures sur l'élaboration de situations d'apprentissage et sur la notion de problème ont précédé les discussions que nous analysons dans cette étude. En outre, nous avons exploré le contenu des objectifs mathématiques minimaux du primaire. Une discussion portant sur la phase de préparation d'une situation d'apprentissage a permis aux futurs maîtres de se familiariser avec la modalité de l'apprentissage coopératif. Ces discussions permettaient ensuite un point d'ancrage pour la suite du cours de didactique.

Les futurs maîtres étaient libres de nous remettre les feuilles sur lesquelles ils avaient inscrit leurs réponses. Toutes les questions étaient inscrites sur une même feuille dans un tableau. Quatre-vingt-huit futurs maîtres ont accepté de nous soumettre, par écrit, leur cheminement durant ces discussions. Ces futurs maîtres sont répartis ainsi: vingt (20) ont analysé la première activité d'enseignement, vingt-trois (23), la deuxième activité d'enseignement, vingt-trois (23), la troisième activité d'enseignement et vingt-deux (22), la quatrième activité d'enseignement.

Ces écrits ont servi à l'analyse des données, analyse réalisée en utilisant la méthode des catégories émergentes (Strauss \& Corbin, 1990). Ainsi, nous avons d'abord regroupé les écrits en attribuant une lettre $(\mathrm{A}, \mathrm{B}, \mathrm{C}, \mathrm{D})$ à chacune des activités d'enseignement, puis à chacun des futurs maîtres (ex. A-1 ou C-3). Nous avons ensuite isolé chacune des activités d'enseignement. Nous avons distingué les discussions réalisées dans les groupes de base de celles des groupes d'experts. Nous avons regroupé les forces et les faiblesses identifiées par les futurs maîtres en unités de sens et ce, pour les groupes de base et pour les groupes 
d'experts. Nous avons enfin identifié des unités de sens pour chacune des situations en ce qui concerne les trois autres questions. Après avoir constaté que plusieurs unités de sens se répétaient pour ces trois questions, nous avons regroupé les quatre activités d'enseignement pour analyser les réponses obtenues à ces trois questions.

En conclusion, cette étude rapporte l'analyse d'une situation de formation vécue dans une classe de didactique. En effet, il nous a semblé qu'une étude approfondie des propos tenus par les futurs maîtres au moment d'un apprentissage pourrait être riche de retombées sur nos dispositifs d'enseignement.

\section{ANALYSE DES ACTIVITÉS D'ENSEIGNEMENT DISCUTÉES EN MILIEU UNIVERSITAIRE}

Nous avons choisi de présenter l'analyse de chacune des pratiques d'enseignement d'abord au moment de la discussion en groupe de base, puis au moment de la discussion en groupe d'experts. La présentation successive de ces deux moments permettra au lecteur de reconnaître les conceptions qui ont émergé dans les groupes de base de même que l'approfondissement amené par la discussion qui a suivi dans les groupes d'experts. Nous réserverons essentiellement nos interprétations, de même que les forces et les limites de la situation de formation comme telle, dans la section discussion.

\section{Première activité d'enseignement discutée}

Les élèves sont en 3e année. L'objectif visé: familiariser les élèves avec le sens de la multiplication et de la division. Le matériel utilisé est l'argent de Monopoly. L'enseignante donne à chaque élève environ 20 billets de $1 \$$.

L'enseignante pose le problème suivant: À l'école d'Émilie, il y a une grande cafétéria. On y sert un menu du jour. Émilie aime beaucoup manger à la cafétéria. Cette semaine, elle a décidé de manger tous les jours à la cafétéria. Chaque repas coûte $3 \$$. Combien va-t-elle payer cette semaine? Montre comment tu as procédé. Existe-t-il d'autres moyens? Lequel de ces moyens semble le plus rapide? 
Après la première semaine, les prix augmentent. Le menu du jour est maintenant $5 \$$. Émilie décide alors de manger à la cafétéria 3 jours seulement. Combien va-t-elle payer pour ses repas cette semaine? Va-t-elle payer plus que la semaine précédente? Explique.

\section{Discussion}

Rappelons que 20 personnes nous ont remis leurs écrits sur cette activité d'enseignement. L'analyse réalisée nous permet de constater que lors de la discussion en groupe de base, plus de la moitié des futurs maîtres ont identifié comme étant une force le fait d'utiliser du matériel pour représenter la situation. Par exemple, un futur maître écrit: "Les élèves peuvent manipuler l'argent et effectuer la multiplication avec cet outil." Ce résultat confirme l'importance accordée à cette composante, comme nous l'avons constaté lors de l'analyse des discussions portant sur la phase de préparation (DeBlois et al., 1998). Ajoutons que certains d'entre eux doutent de la pertinence d'utiliser l'addition pour résoudre ce problème. Toutefois, cette composante ne retient l'attention que de $20 \%$ des futurs maitres lors de la discussion dans le groupe d'experts. En effet, le débat semble mener la moitié des futurs maitres à attribuer la principale force au fait de revenir sur des connaissances antérieures des élèves et de susciter l'utilisation de plusieurs stratégies. Ainsi, un futur maître écrit: "Obligé de passer par ce qu'ils connaissent pour aller vers la multiplication; [les] questions l'y obligent." Il s'agit sans doute dans ce cas à la fois d'une manifestation de l'apprentissage que nous avons voulu susciter lors de la rencontre précédente et de l'émergence d'une nouvelle prise de conscience.

En ce qui concerne les faiblesses attribuées à cette activité d'enseignement, la majorité des futurs maîtres croient que le fait de ne traiter que d'une partie de l'objectif visé est une faiblesse. En outre, près du tiers des personnes croient que trop de questions sont posées. Un futur maître explique ce choix en répondant: "Existe-t-il d'autres moyens de faire et comment? L'enfant va-t-il être capable de trouver d'autres moyens? Trop de questions." Le débat en groupe d'experts ne modifie pas ces dernières conceptions de façon notable. En effet, le fait de ne pas traiter de la division et de limiter le matériel à utiliser est 
retenu par près de $75 \%$ d'entre eux. Le nombre de personnes qui voient le questionnement comme une faiblesse a toutefois diminué. Devons-nous interpréter ces résultats comme une recherche de conformité à la formulation des objectifs du programme d'études? Nous reconnaissons plutôt, dans ce cas, un moment propice pour le formateur qui souhaite attirer l'attention des futurs maîtres sur les implications d'une stratégie d'enseignement comme la résolution de problème et les programmes d'études. Le tableau 1 regroupe les résultats obtenus.

Tableau 1

Analyse des conceptions pour la première activité d'enseignement

\begin{tabular}{|c|c|c|c|c|}
\hline & Groupes de bas & & Groupes d'expert & \\
\hline Forces & $\begin{array}{l}\text { Problème répresente } \\
\text { par du matériel: } \\
\text { Questionnement } \\
\text { amène l'exploration: } \\
\text { Commutativité: } \\
\text { Ensemble des } \\
\text { éléments précédents: } \\
\text { Clartédu problème: } \\
\text { Total des personnes: }\end{array}$ & $\begin{array}{r}12 \\
4 \\
2\end{array}$ & $\begin{array}{l}\text { Connaissances } \\
\text { antérieures et } \\
\text { développement de } \\
\text { stratégies: } \\
\text { Commutativité } \\
\text { amenée par le } \\
\text { questionnement: } \\
\text { Problème représente } \\
\text { par le matériel: } \\
\text { Total des personnes: }\end{array}$ & $\begin{array}{r}4 \\
20\end{array}$ \\
\hline Faiblesses & $\begin{array}{l}\text { Ne traite pas } \\
\text { l'objectif visé: } \\
\text { Trop de questions: } \\
\text { Le matériel est limité: } \\
\text { Aucune: } \\
\text { Total des personnes: }\end{array}$ & $\begin{array}{r}12 \\
6 \\
1 \\
1 \\
20\end{array}$ & $\begin{array}{l}\text { Ne traite pas } \\
\text { l'objectif visé: } \\
\text { Le matériel est limité: } \\
\text { Trop de questions: } \\
\text { Aucune: } \\
\text { Total des personnes: }\end{array}$ & $\begin{array}{r}10 \\
6 \\
3 \\
1 \\
20\end{array}$ \\
\hline
\end{tabular}




\section{Deuxième activité d'enseignement discutée}

Les élèves sont en 4e année. L'objectif visé: se familiariser avec le sens de la multiplication et de la division. Chaque élève a une centaine de jetons.

L'enseignante présente aux élèves le problème suivant:

Vous êtes à une exposition. Pour gagner un jeu, vous devez avoir 36 jetons. Vous êtes 5 amis. Vous décidez de regrouper vos jetons. Chaque enfant a 6 jetons. Combien de jetons avez-vous en tout? Comment procédez vous? Existe-t-il d'autres moyens? Lequel de ces moyens semble le plus rapide? Avez-vous suffisamment de jetons pour les échanger contre le jeu? Combien manque-t-il de jetons? Que ferezvous pour obtenir le jeu?

Maintenant, tu as 30 jetons. Tu les distribues à tes amis de façon que chacun en reçoive 6 . À combien d'amis peux-tu distribuer tes jetons? Comment procèdes-tu? Existe-t-il d'autres moyens? Lequel de ces moyens semble le plus rapide?

\section{Discussion}

Les vingt-trois (23) futurs maîtres ayant discuté de cette activité d'enseignement en groupe de base attribuent de nouveau, majoritairement, l'utilisation de matériel comme étant la principale force. La discussion en groupes d'experts semble toutefois les amener à revoir cette position pour s'attarder plus particulièrement à la compréhension de l'opération de multiplication. Par exemple, un futur maître précise la relation qu'il a reconnue entre la multiplication et la division en écrivant: "Problèmes en relation avec les objectifs. Les deux problèmes se complètent. Les propriétés de la $\mathrm{x}$ et de la $\div$ ".

Pour ce qui est des faiblesses, à nouveau près des deux tiers des futurs maîtres croient que le nombre de questions planifiées est problématique. "Trop de questions dans le problème. L'élève peut être déstabilisé," écrit un futur maître. Deux explications semblent apparaître. D'une part, la formulation de questions déstabilise l'élève. D'autre part, susciter une déstabilisation des connaissances de l'élève 
revient à placer ce dernier dans une situation non souhaitable. Cette conception demeure lors de la discussion dans le groupe d'experts. Le tableau 2 donne l'ensemble des résultats obtenus.

\section{Troisième activité d'enseignement discutée.}

L'activité concerne la classe de 5e année. L'objectif visé est de familiariser les élèves avec le sens de la multiplication et de la division. Les élèves peuvent utiliser des crayons de couleur pour illustrer les situations.

L'enseignante présente aux élèves le problème suivant:

Trois garçons et quatre filles veulent danser. Chaque garçon veut danser avec chaque fille et chaque fille avec chaque

Tableau 2

Analyse des conceptions pour la deuxième activité d'enseignement

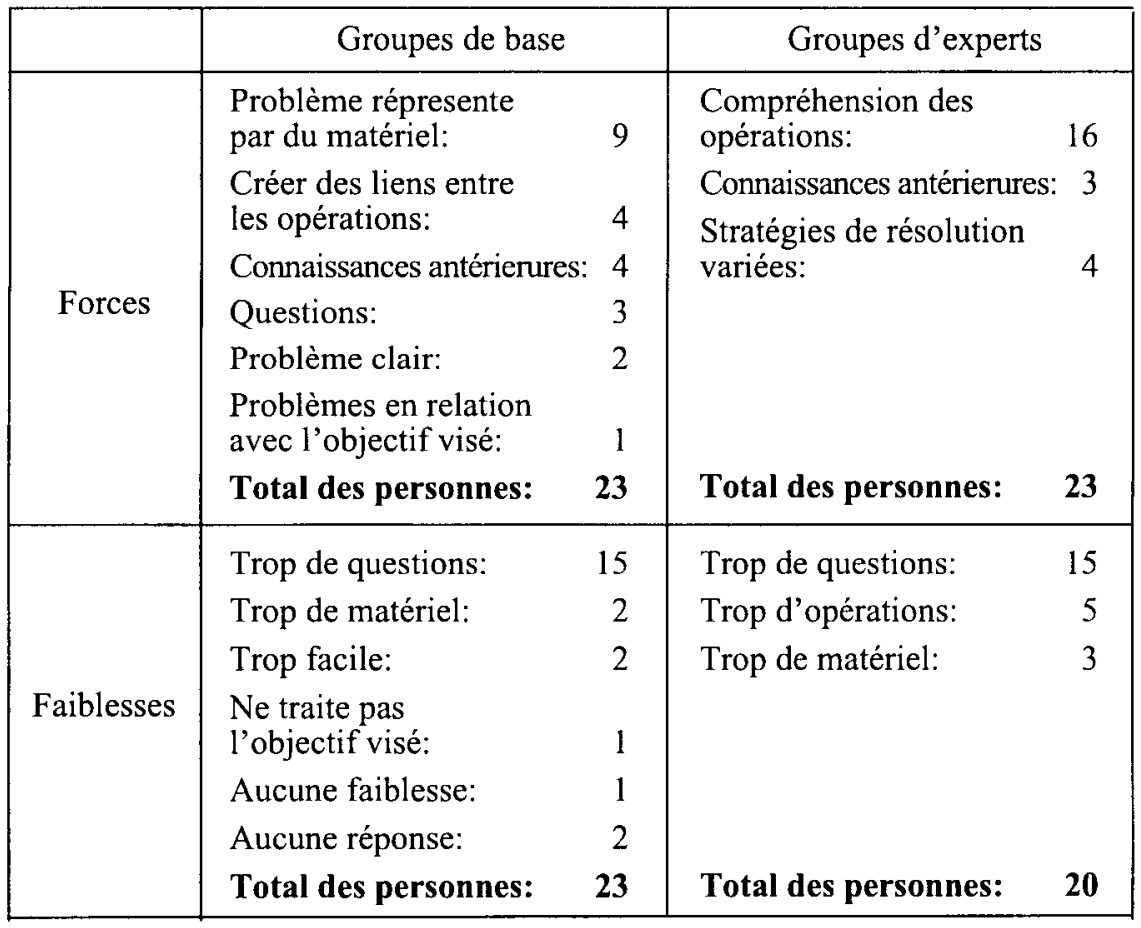


garçon. Combien y a-t-il de couples possibles? L'enseignante invite les élèves à imaginer un dessin qui pourrait décrire la situation. Montre comment tu as procédé. Existe-t-il d'autres moyens? Lequel de ces moyens semble le plus rapide?

\section{Discussion}

Durant la discussion en groupe de base, plus de $80 \%$ des vingt-trois (23) futurs maîtres qui se sont attardés à cette activité attribuent la principale force à l'utilisation du dessin pour représenter le problème. Par exemple, un futur maître écrit: "Dessiner la situation facilitera la compréhension." Il s'agit donc, de nouveau, d'un intérêt marqué pour l'utilisation de matériel. La discussion en groupe d'experts confirme cette position sans toutefois que cette tendance ne soit aussi importante que dans les deux premières activités d'enseignement. De nouvelles unités de sens émergent. Moins de la moitié des futurs maîtres semblent prendre conscience que les élèves pourraient présenter des stratégies de résolution variées et que les élèves pourraient trouver eux-mêmes. Ainsi, une fois que le produit cartésien est reconnu comme étant un des sens de la multiplication, la discussion pourrait permettre aux futurs maîtres de reconnaître que la diversité des procédures réalisées par les élèves devient une force. Un futur maître écrit: "Petit nombre utilisé. Illustration du problème. Permet de voir plusieurs façons de faire le problème." Dans le cas où ces deux thèmes seraient regroupés, nous pourrions conclure que le groupe d'experts a permis de discuter de l'activité d'enseignement en prenant en compte l'importance de l'activité de l'élève, mais pour moins de la moitié de ces futurs maitres.

La principale faiblesse de cette activité d'enseignement est attribuée, par plus de $75 \%$ des futurs maîtres, au fait de ne pas traiter la division. Notons que parmi eux, certains ne voient pas d'opération de multiplication au moment de la discussion dans le groupe de base, comme nous nous y attendions. Ainsi, un futur maître écrit: "Pas de présence de la division et mauvais exemple pour la multiplication." Très peu de personnes ont soulevé le fait qu'il y ait plusieurs questions. Ces résultats se sont cristallisés autour du thème "ne traite pas de l'objectif visé" durant la discussion en groupe d'experts. Le tableau 3 donne le détail des résultats obtenus. 
Tableau 3

Analyse des conceptions pour la troisième activité d'enseignement

\begin{tabular}{|c|c|c|c|c|}
\hline & \multicolumn{2}{|l|}{ Groupes de base } & \multicolumn{2}{|l|}{ Groupes d'experts } \\
\hline \multirow{7}{*}{ Forces } & Illustrer le problème & & Illustrer par un dessin: & 12 \\
\hline & par un dessin: & 20 & Les élèves trouvent & \\
\hline & Questionnement: & 1 & eux-mêmes: & 3 \\
\hline & Connaissances antérieures: & 1 & Stratégies de résolution & \\
\hline & Les élèves trouvent & & variées: & 7 \\
\hline & eux-mêmes: & 1 & Connaissances antérieures: & 1 \\
\hline & Total des personnes: & 23 & Total des personnes: & 23 \\
\hline \multirow{6}{*}{ Faiblesses } & $\begin{array}{l}\text { Ne traite pas } \\
\text { l'objectif visé: }\end{array}$ & 14 & $\begin{array}{l}\text { Ne traite pas } \\
\text { l'objectif visé: }\end{array}$ & 23 \\
\hline & Trop de questions: & 2 & & \\
\hline & $\begin{array}{l}\text { Trop facile our } \\
\text { trop difficile: }\end{array}$ & 3 & & \\
\hline & Carté de l'énoncé & 1 & & \\
\hline & Aucune: & 3 & & \\
\hline & Total des personnes: & 23 & Total des personnes: & 23 \\
\hline
\end{tabular}

\section{Quatrième activité d'enseignement à discuter (contre-exemple)}

L'activité se déroule en $4 \mathrm{e}$ année. L'objectif visé est de susciter la compréhension du sens de la division. L'enseignante privilégie les jetons comme matériel.

L'enseignante pose le problème suivant: Trois enfants partagent 15 pommes. Combien de pommes chaque enfant reçoit-il?

L'enseignante prend ensuite 15 jetons et fait un premier ensemble de 3 jetons, ensuite un autre de 3 autres jetons et ainsi de suite. Elle compte ensuite le nombre de groupements de 3 jetons. Elle trouve 5 . Elle donne aux élèves le même problème avec les nombres suivants: 60 et 4 . 


\section{Discussion}

De nouveau, la discussion en groupe de base amène $75 \%$ des vingtdeux (22) futurs maîtres à considérer l'utilisation d'un matériel comme une force. En outre, ils attribuent une force au fait que l'enseignante offre une démonstration de la procédure aux élèves. Un futur maître écrit: "La modélisation, la visualisation offerte par l'enseignante." Toutefois, seulement la moitié d'entre eux reconnaissent une confusion entre le sens partage du problème et le sens mesure de la manipulation offerte par l'enseignante. Cela ne nous surprend guère, comme nous l'avons déjà évoqué. La discussion en groupes d'experts semble les sensibiliser à cette erreur, ce qui montre l'apport de cette modalité d'intervention dans l'approfondissement des contenus à enseigner. "L'enseignante fait la démonstration avec des ensembles de 3, alors que le problème suggère plutôt de répartir les éléments en 3 groupes (de 5 , évidemment) et non 5 groupes de 3 éléments (pommes)." écrit un futur maître. Toutefois, aucun nouveau thème n'émerge de cette deuxième discussion. Il semble donc que la clarification du contenu mathématique ait occupé tout l'espace et tout le temps de discussion. Le tableau 4 rapporte l'ensemble des catégories qui ont émergé de l'analyse.

Tableau 4

Analyse des conceptions pour la quatrième activité d'enseignement

\begin{tabular}{|c|lr|lr|}
\hline & \multicolumn{2}{|c|}{ Groupes de base } & \multicolumn{2}{c|}{ Groupes d'experts } \\
\hline \multirow{5}{*}{ Forces } & Problème représenté & & Problème représenté & \\
& par le matériel: & 16 & par le matériel: & 20 \\
& Démonstration: & 5 & Rien: & 2 \\
& Grandeur des nombres: & 1 & & \\
& Total des personnes: & $\mathbf{2 2}$ & Total des personnes: & $\mathbf{2 2}$ \\
\hline \multirow{5}{*}{ Faiblesses } & Confusion de sens: & 10 & Confusion de sens: & 16 \\
& Faire une démonstration: & 5 & Démonstration et & \\
& Grandeur des nombres: & 3 & confusion de sens: & 6 \\
& Matériel choisi: & 4 & & \\
& Total des personnes: & $\mathbf{2 2}$ & Total des personnes: & 22 \\
\hline
\end{tabular}




\section{Synthèse des forces et des faiblesses pour les quatre activités d'enseignement}

En réalisant une synthèse de ces résultats, nous constatons que les discussions en groupe de base amènent $65 \%$ des futurs maîtres à considérer comme une force le fait d'illustrer la situation avec un matériel ou un dessin. Un ensemble d'autres thèmes émergent de façon moins importante: le questionnement offert aux élèves, l'importance à accorder aux connaissances antérieures, les actions posées sur des ensembles d'objets pour introduire la multiplication, la création de relations entre les opérations de multiplication et de division. Ces résultats donnent le portrait d'une première conception à l'égard des scénarios d'enseignement proposés.

Les discussions en groupes d'experts semblent enrichies par de nouveaux thèmes. Toutefois, dans le cas de la troisième et de la quatrième activité d'enseignement ces nouveaux thèmes n'apparaissent pas. Il se peut que la discussion n'ait porté que sur ce qui posait problème: le sens produit cartésien pour la troisième activité d'enseignement et la présence des deux sens de la division dans la quatrième activité d'enseignement. Ces confusions liées au contenu mathématique ont été résolues sans permettre simultanément d'approfondir le thème relatif à l'utilisation du matériel. En ce qui concerne les deux premières activités d'enseignement, de nouvelles unités de sens émergent, plus particulièrement en ce qui concerne l'activité de l'élève: les connaissances antérieures des élèves et le développement des stratégies de résolution par les élèves. Ces deux thèmes ont été regroupés car ils apparaissent souvent simultanément pour près du tiers d'entre eux. Le développement de la compréhension de concepts retient l'attention de moins d'un cinquième des futurs maîtres. Le tableau 5 donne les résultats obtenus.

En somme, l'apport d'une deuxième discussion laisse apparaître de nouvelles conceptions relativement à une activité d'enseignement mettant en scène les concepts de multiplication et de division: la compréhension tient une place en mathématiques, l'illustration d'un problème pourrait faciliter cette compréhension, cette illustration 
Tableau 5

Synthèse de forces identifiées par l'ensemble des futurs maîtres ${ }^{3}$

\begin{tabular}{|c|c|c|c|}
\hline Groupes de base & & \multicolumn{2}{|l|}{ Groupes d'experts } \\
\hline $\begin{array}{l}\text { Problème représenté par } \\
\text { un matériel: }\end{array}$ & $65 \%$ & $\begin{array}{l}\text { Problème représenté par } \\
\text { le matériel: }\end{array}$ & $41 \%$ \\
\hline Questionnement: & $9 \%$ & Connaissances antérieures & \\
\hline Connaissances antérieures: & $6 \%$ & et développement de & $30^{\circ}$ \\
\hline Démonstration: & $6 \%$ & Comnréhension des & sor \\
\hline $\begin{array}{l}\text { Créer des liens entre } \\
\text { les opérations: }\end{array}$ & $5 \%$ & & $18 \%$ \\
\hline Clarté du problème: & $3 \%$ & eux-mêmes: & $3 \%$ \\
\hline Commutativité: & $2 \%$ & Commutativité amené & \\
\hline $\begin{array}{l}\text { Problèmes en relation } \\
\text { avec l'objectif: }\end{array}$ & $1 \%$ & $\begin{array}{l}\text { questionnement: } \\
\text { Aucune: }\end{array}$ & $\begin{array}{l}5 \% \\
2 \%\end{array}$ \\
\hline Grandeur des nombres: & $1 \%$ & & \\
\hline Ensemble d'éléments: & $1 \%$ & & \\
\hline
\end{tabular}

faciliterait l'émergence des connaissances antérieures et le développement de stratégies de résolution chez les élèves. Rappelons que ces nouvelles conceptions n'apparaissent que dans le cas où les activités d'enseignement ne posent pas de problème de contenu mathématique. D'autre part, la rencontre qui a précédé cette étude a suscité une discussion sur la phase de préparation, phase dans laquelle un accent est mis sur le thème "connaissances antérieures des élèves". L'étude du tableau 5 montre que ce thème a été retenu par $6 \%$ des futurs maîtres dans les groupes de base et par $30 \%$ d'entre eux dans les groupes d'experts. Il est possible que les futurs maîtres cherchent à intégrer au débat les connaissances nouvellement acquises.

Deux aspects sont à éviter au moment de vivre des activités d'enseignement pour les futurs maîtres: l'absence de conformité à l'objectif visé $(28 \%)$ et le fait de poser des questions aux élèves $(26 \%)$. $\mathrm{Si}$ la discussion en groupe d'experts a pu provoquer un enrichissement au plan du contenu mathématique, elle n'a pas permis d'approfondir ni de remettre en question les faiblesses déjà identifiées. Ainsi, 38\% des 
futurs maitres considèrent toujours comme une faiblesse le fait de ne pas avoir traité, en tout ou en partie, de l'objectif visé alors que $20 \%$ des futurs maîtres croient toujours que trop de questions sont posées. Nous pourrions poser deux hypothèses à l'égard de ces résultats. La conformité aux objectifs pourrait être le reflet de l'importance à réaliser un enseignement correct du point de vue des futurs maîtres. En outre, il est possible que certains futurs maitres aient une intuition selon laquelle le questionnement peut être vu comme une façon de guider les élèves. Toutefois, ils ne sont pas en mesure d'articuler une argumentation qui pourrait influencer l'ensemble des futurs maîtres. Le tableau 6 illustre l'ensemble des résultats obtenus.

En conclusion, il semble qu'une phase de réalisation, où un problème suivi de questions est proposé, laisse émerger une conception selon laquelle le questionnement peut poser problème aux élèves: il est donc considéré indésirable. En outre, nous pourrions retenir que, pour eux, le respect d'un objectif correspond à leur conception d'une bonne activité d'enseignement.

Tableau 6

\section{Synthèse de faiblesses identifiées pour l'ensemble des futurs maîtres}

\begin{tabular}{|lr|lc|}
\hline \multicolumn{2}{|c|}{ Groupes de base } & \multicolumn{2}{c|}{ Groupes d'experts } \\
\hline Ne traite pas de l'objectif: & $31 \%$ & Ne traite pas de l'objectif: & $38 \%$ \\
Trop de questions: & $26 \%$ & Trop de questions: & $20 \%$ \\
Confusion de sens: & $11 \%$ & Confusion de sens: & $18 \%$ \\
Trop ou limite de matériel: & $7 \%$ & Matériel limité ou trop & \\
Trop facile our trop difficile: & $6 \%$ & de matériel: & $10 \%$ \\
Faire une démonstration: & $6 \%$ & Démonstration et & $7 \%$ \\
Grandeur des nombres: & $3 \%$ & confusion de sens: & $6 \%$ \\
Aucune réponse: & $6 \%$ & Trop d'opérations: & $6 \%$ \\
Aucune: & $3 \%$ & Aucune: & $1 \%$ \\
Clarté de l'énoncé: & $1 \%$ & & \\
\hline
\end{tabular}




\section{Ces quatre activités d'enseignement suscitent chez les élèves...}

Nous avons demandé aux futurs maîtres, en groupes de base, ce qu'ils croient possible de développer chez les élèves en proposant de telles situations d'apprentissage. Près de $75 \%$ d'entre eux reconnaissent que le développement d'une compréhension des concepts mathématiques est suscité. Par exemple, un futur maître écrit: "Des habiletés à travailler avec du matériel pour concevoir le concept de division (ou autre opération). De leur faire voir que la division peut se travailler avec du concret au semi-concret. Ainsi, des habiletés à imaginer dans sa tête la situation avec la manipulation du problème." Parmi ces derniers, $15 \%$ croient que nous cherchons à susciter l'évolution de la compréhension des ensembles vers les opérations comme l'explique l'un d'eux: "Le concept d'ensemble pour enseigner la multiplication et en venir au concept de division. Addition répétée." Enfin, 17\% des futurs maîtres croient que nous suscitons le développement de stratégies de résolution chez les élèves. Ainsi, l'un d'eux écrit: "Capacité de trouver des stratégies pour résoudre plus facilement les divisions." Le tableau 7 donne un aperçu de l'ensemble des résultats.

\section{Tableau 7}

\section{Identification de l'apport des activités d'enseignement}

Le développement d'une compréhension des concepts mathématiques $\quad 59 \%$

L'évolution des ensembles vers les opérations $\quad 16 \%$

Le développement de stratégies de résolution chez les élèves $\quad 17 \%$

La création de liens entre le vécu des élèves et les mathématiques $\quad 4 \%$

La notion d'ensembles $\quad 3 \%$

Pas de réponse $\quad 1 \%$ 


\section{Que retenez-vous pour l'enseignement de cette notion?}

Cette question visait à identifier comment les futurs maîtres conçoivent leur rôle dans la classe. Nous leur avons posé la question suivante: "Que retenez-vous pour l'enseignement de cette notion?" Le tiers des personnes ont répondu qu'ils accorderont une importance aux relations à créer entre les opérations. Un futur maître s'exprime ainsi: "La multiplication et la division doivent être traitées parallèlement." Vingt-huit pour cent écrivent qu'elles se préoccuperont de l'aspect concret en utilisant soit un matériel soit le vécu des élèves. Les autres réponses se distribuent entre un enseignement vu dans une perspective plus constructiviste (guider les élèves par des questions, se préoccuper des connaissances antérieures des élèves ou placer les élèves en activité $21 \%$ ) et, dans une moindre mesure, un enseignement vu comme une démarche d'explication (la clarté des propos à tenir aux élèves et les explications à leur donner $11 \%$ ). Il semble donc que plus de la moitié des futurs maîtres se préoccupent d'abord du contenu mathématique avant de s'attarder à des principes d'enseignement. Le tableau 8 regroupe les informations recueillies.

Tableau 8

Conceptions du rôle de l'enseignant our de l'enseignante

Créer des liens entre les opérations $33 \%$

Le matériel ou le vécu des élèves $\quad 28 \%$

La clarté des propos et des explications à donner aux élèves $\quad 11 \%$

Guider par des questions $\quad 10 \%$

Connaissances antérieures $\quad 6 \%$

Ensemble des précautions $\quad 5 \%$

Placer les élèves en activités $\quad 5 \%$

Se concentrere sur les notions plutôt que sur la situation $\quad 1 \%$

Rien $2 \%$ 
En somme, seulement $26 \%$ des futurs maîtres conçoivent leur rôle dans une perspective constructiviste. En nous référant aux thèmes relatifs à la création de relations entre les opérations et entre le vécu des élèves, nous constatons que $61 \%$ d'entre eux s'attardent au sens à donner aux concepts mathématiques. La conception qui veut qu'enseigner corresponde à expliquer n'est retenue que par $11 \%$ des futurs maîtres.

\section{Que retenez-vous pour l'apprentissage de cette notion?}

La dernière question visait à identifier comment les futurs maîtres conçoivent le rôle des élèves. Nous leur avons posé la question suivante: “Que retenez-vous pour l'apprentissage de cette notion?" De nouveau, l'utilisation du matériel (38\%) émerge de façon importante, suivi de près par l'identification des connaissances antérieures pertinentes chez les élèves $(28 \%)$. Nous croyons que la manifestation d'un intérêt à reconnaître les connaissances antérieures des élèves pourrait devenir une entrée pour la mise en œuvre d'un questionnement. Toutefois, l'exploration de la part de l'élève, exploration qui permettrait à ce dernier de développer de nouvelles stratégies, ne retient explicitement l'attention que de $19 \%$ des futurs maîtres, ce qui nous semble paradoxal. Il est possible que le fait de mentionner l'importance de l'utilisation du matériel implique, pour eux, une exploration de la part des élèves. Le tableau 9 regroupe l'ensemble des résultats obtenus.

Tableau 9

Conceptions des rôles de l'élève

Le matériel $38 \%$

Connaissances antérieures chez les élèves $28 \%$

Choix des stratégies et l'exploration de la part de l'élève $19 \%$

Créer des liens entre les opérations $6 \%$

Leçons doivent être simples et concrètes $3 \%$

Questions à formuler $3 \%$

Croient que ces concepts son plus longs à acquérir que + et $1 \%$

Pas de réponse $2 \%$ 
Ainsi, il semble que l'ensemble des futurs maîtres se préoccupe essentiellement de l'utilisation du matériel. L'identification des thèmes, connaissances antérieures et exploration des élèves pour susciter l'apprentissage de cette notion, pourrait être interprété comme une manifestation des rôles de l'enseignant et de l'élève simultanément. L'émergence de ces deux pôles, au même moment, pourrait générer une tension cognitive chez les futurs maîtres.

\section{DISCUSSION ET IMPLICATIONS PÉDAGOGIQUES}

Nous nous sommes posés les questions suivantes: Quelles conceptions les futurs maîtres explicitent-ils au moment d'une discussion sur une phase de réalisation? Puisque, au moment de la discussion sur une phase de préparation les futurs maîtres croient qu'il est souhaitable de limiter les difficultés à présenter, comment le concept de résolution de problèmes est-il compris à titre de stratégie d'enseignement? Comment réagissent-ils à une planification qui présente des questions plutôt qu'une démarche d'explication linéaire?

Nos résultats laissent apparaître certaines conceptions à l'égard de ce type d'activité d'enseignement. Dans un premier temps, comme cela a été le cas chez les enseignants et les enseignantes en exercice (Bednarz, 2000), les futurs enseignants s'attardent au contenu mathématique. L'illustration du problème par un matériel tient alors une place importante. Les discussions en groupe d'experts ne semblent susciter un approfondissement que dans le cas où le contenu mathématique ne pose plus de problème aux futurs maîtres. À ce moment, leur attention est attirée par le développement de la compréhension chez les élèves.

Les discussions en groupe d'experts n'ont pas permis de remettre en question les faiblesses déjà identifiées comme l'absence de conformité aux objectifs d'apprentissage ou le questionnement. Les discussions en groupe d'experts ont laissé apparaître une utilisation stricte de la situation d'apprentissage en regard de l'objectif à traiter. Il semble donc y avoir une recherche de conformité au curriculum qui pourrait créer une première tension chez les futurs maîtres au moment d'une expérience en classe. En effet, une stratégie d'intervention par la résolution de 
problèmes nécessite d'interpréter les objectifs en les regroupant, éventuellement, différemment de la façon dont ils sont élaborés dans les programmes d'études. Attardons-nous davantage au questionnement.

Thompson (1984) révèle que les croyances des futurs maîtres du primaire sont semblables à celles des "mathophobes." Nous expliquerions leur appréhension différemment. Les discussions semblent avoir conduit les futurs maîtres à concevoir leur rôle comme étant celui qui permet aux élèves de créer des relations entre les opérations. Rappelons que plus de la moitié des futurs maîtres se préoccupent d'abord du contenu mathématique avant de s'attarder à des principes d'enseignement. Cette conception pourrait les conduire à entretenir un mythe: l'enseignant doit détenir toutes les réponses. Que signifie détenir toutes les réponses? Les réponses aux questions de contenu disciplinaire ou les interventions par rapport à une procédure imprévue de l'élève. Un glissement de sens apparaîtrait entre le contenu à enseigner et le "comment"enseigner. En effet, le "comment" enseigner devient une réponse à une question professionnelle. Les futurs maîtres exprimeraient donc plutôt une insécurité par rapport aux réactions à adopter devant une réponse imprévue d'un élève. En effet, que faire avec la réponse de l'élève? Répondre à cette question, c'est concevoir l'apprentissage comme un processus.

Toutefois, les futurs maîtres expriment plutôt une conception "magique" de l'apprentissage. En effet, l'élève utilise du matériel et laisse émerger les connaissances antérieures pertinentes à la résolution du problème. L'identification de ces thèmes pourrait être interprétée comme une assimilation des rôles de l'élève à ceux de l'enseignant. L'émergence de ces deux pôles, au même moment, pourrait générer une deuxième tension chez les futurs maîtres puisqu'ils ne distinguent pas vraiment le rôle de l'élève de celui de l'enseignant.

Carpenter et Fenema (1989) avaient constaté comment une connaissance approfondie de la pensée des élèves permettait aux enseignants de les questionner et d'être à l'écoute de leurs réponses. Il semble que les futurs maîtres cheminent plutôt à partir de la façon dont ils imaginent les manipulations que les élèves réalisent avec du matériel. Ainsi, à partir d'une discussion portant sur l'illustration d'un problème 
émergeraient les thèmes relatifs au développement de la compréhension des élèves (connaissances antérieures des élèves et au développement de stratégies de résolution facilitant une compréhension). Leurs expériences d'élèves pourraient avoir une grande influence dans cette démarche.

L'hypothèse selon laquelle des discussions entre des futurs maîtres sur des activités d'enseignement déjà planifiées les conduiraient à questionner leur conception de l'enseignement des mathématiques semble confirmée en partie. En effet, nous avons pu reconnaître l'apport de la discussion en groupe d'experts à certaines conditions. Pour chacune des activités d'enseignement, la lecture des tableaux nous permet de constater qu'un thème a été privilégié: l'utilisation du matériel. Les futurs maîtres ont ensuite exploré, en groupe d'experts, d'autres thèmes comme la diversité des procédures des élèves, puis la nécessité de l'activité des élèves, mais seulement dans le cas où le contenu mathématique ne poserait pas de problème. Ces résultats confirment donc l'hypothèse de Wideen, Mayer-Smith et Moon (1998) qui reconnaissaient l'influence de l'apprentissage coopératif et du jeu entre les concepts présents dans les problèmes proposés. Toutefois, ces discussions ont des limites à deux moments. En effet, dans le cas où le contenu mathématique devrait être clarifié et dans celui où l'attention des futurs maîtres est attirée sur les faiblesses d'une activité d'enseignement, les conceptions semblent se cristalliser.

Les moments de tension identifiés pourraient nous guider au moment de formuler des implications pédagogiques. La recherche de conformité au curriculum qui peut créer un conflit avec une stratégie d'intervention par la résolution de problèmes, de même que l'assimilation des rôles de l'élève à ceux de l'enseignant, nous invitent à préciser les questions à proposer aux futurs maîtres durant leurs discussions. Ainsi, il semble préférable de susciter des discussions sur ce qu'ils considèrent comme une force: l'utilisation d'un matériel didactique et sa pertinence dans le développement de la compréhension de l'élève. En effet, une discussion portant sur les faiblesses n'apporte pas les résultats escomptés. Ce n'est que par la suite que l'étude des procédures et des réflexions d'un élève qui présente des difficultés deviendrait nécessaire à la réalisation de l'activité d'enseignement dans 
une classe. Cette étude de la pensée de l'élève pourrait leur permettre, dans un premier temps, de distinguer le rôle qu'ils auront à jouer de celui qu'ils ont toujours joué. Dans un deuxième temps, cette étude pourrait les sécuriser devant des réactions imprévues d'élèves. Le questionnement pourrait être vu différemment. Toutefois, ces discussions devront être complétées afin d'amener les futurs maîtres à cerner les implications d'une telle modalité d'intervention plus particulièrement sur le plan des contraintes d'une classe.

En nous intéressant à l'explicitation des conceptions des futurs maîtres du primaire au début de leur formation, nous avons voulu comprendre comment ils conçoivent la résolution de problèmes comme stratégie d'intervention. Une telle explicitation nous permet d'apporter des éléments de réponses aux questions relatives à la construction de savoirs et de pratiques à l'université. En effet, une explicitation des conceptions apporte certaines réponses à la question du type de didactique à présenter à l'université. Une didactique qui conduit les futurs maîtres à interpréter ce qui se passe dans une classe pour créer leurs interventions.

\section{Les notes}

1 Dans le cadre de cette étude, l'expesssion conception est ici privilégiée à celle de représentation comme le porposent Jonnaert et Van der Borght (2000). En effet, la notion de représentation peut être confondue avec celle de représentations sociales. Pour cette raison, les idées et les croyances des futurs maîtres relativement aux concepts mathématiques, mais aussi aux idées et aux croyances relativement à leur enseignement et leur apprentissage seront intégrées à l'intérieur du vocable conception.

2 Nous avons choisi de présenter les quatre activités d'enseignement dans la section analyse afin d'éviter des redondances et de faciliter la lecture et la compréhension des analyses sans devoir retourner sans arrêt à la section méthode.

3 En transformant nos résultats en pourcentage, nous avons complété à l'unité lorsque nous obtenions 0,5 , ce qui explique que certains tableaux donnent un total de oo\$ plutôt que $100 \%$. 


\section{Références}

Bednarz N., Gattuso L., \& Mary, C. (1997). Changes in student teacher views of the mathematics teaching/learning process at the secondary school. In Proceedings of the 20th Conference of the International Group for the Psychology of Mathematics Education, Tome 2, 59-66. University of Valencia, Spain.

Bednarz N. (2000). Formation continue des enseignants en mathématique: Une nécessaire prise en compte du contexte. Dans Des didacticiens se rencontrent. Collection Astroïde, Modulo. Montréal, QC.

Benden B.M., \& Wilson M.R. (1996). Developing problem-solving conceptions of mathematics: A preservice teacher's experiences. In Proceedings of the North American Chapter of the International Group for the Psychology of Mathematics Education, Tome 2. Panama City, FL, 251-257.

Boero P., Dapueto, C., \& Parenti L. (1996). Didactics of mathematics and the professional knowledge of teachers. International Handbook of Mathematics Education, Tome 2, 1097-1122.

Britzman D.P. (1991). Practice makes practice. New York, NY: University of New York Press.

Brousseau, G. (1986). Fondements et méthodes de la didactique des mathématiques. Recherches en didactique des mathématiques, 7(2), 33-115.

Carpenter T.P., \& Fenama E. (1989). Building on the knowledge of students and teachers. Proceeding of the 13e International Group ofr the Psychology of Mathematics Education, Paris, (1), 34-45.

Chapman, O. (1996). Reconstruction teachers' knowledge of intervention in teaching problem solving. In In Proceedings of the North American Chapter of the International Group for the Psychology of Mathematics Education, Tome 2. Panama city, FL, 263-268.

Civil, M. (1993). Prospective elementary teachers' thinking about teaching mathematics. Journal of Mathematical Behavior, 12, 79-109.

DeBlois, L., \& Squalli, H. (1997). L'analyse des erreurs des élèves en mathématiques par des étudiantes et des étudiants en formation initiale à l'enseignement. In H. Ziarko \& M. Tardif (Eds.), La formation initiale continuités et ruptures, (pp. 125-143). Québec, QC: Presses de l'Université Laval.

DeBlois, L., Uwimana, A., \& Vézina, N. (1998). De nos pratiques d'enseignement vers une compréhension de l'enseignement des mathématiques. In Actes du colloque de la 4e Biennale sur la Formation et l'Éducation. Paris: Sorbonne. 
Dionne, J. (1988). Vers un renouvellement de la formation et du perfectionnement des maîtres du primaire: le problème de la didactique des mathématiques. Montréal, QC: Université de Montréal, Faculté des sciences de l'éducation, Collection Prix Grégoire.

Emenaker, C.E. (1995). The influence of a problem-solving approach to teaching mathematics on preservice teachers' mathematical beliefs. Washington, DC: ERIC no. ED 391657.

Ernest, P. (1991). The philosophy of mathematics education. London: Falmer Press.

Ernest, P. (1989). The impact of beliefs on the teaching of mathematics. In P. Ernest (dir.), Mathematics Teaching: The State of the Art, (pp. 249-254). New York, NY: Falmer Press.

Fenema, E., Carpenter, T.P., Franke, L.M., Levi, L., Jacobs, V.R., Empson, S.B. (1996). A longitudinal study of learning to use children's thinking in mathematics instruction. Journal for Research in Mathematics Education, 27, 403-434.

Hong, E. (1995). Preservice elementary teachers' conceptions about teaching word problem solving: The effect of methods instruction. Nevada: ERIC ${ }^{\circ}$ ED 388385.

Jaworski, B. (1994). Investigating mathematics teaching. New York, NY: Falmer Press.

Jonnaert \& Van der Borght. (1999). Créer des conditions d'apprentissage. Paris, Bruxelles: Editions DeBoeck.

Johnson, D.W., \& Johnson, R.T. (1988). Cooperation in the classroom. Edina, MN: Interaction Book Company.

Johnston, M. (1990). Teacher's background and beliefs: Influences on learning to teach in the social studies. Theory and Research in Social Education, 18, 207-232.

Miller, C.M. (1996). Teachers as problem solvers/problem solvers as teachers: Teachers' practice and teaching of mathematical problem-solving. In Proceedings of the North American Chapter of the International Group for the Psychology of Mathematics Education. Panama city Fl. Tome 2, 269-274.

Ministère de l'Éducation. (1988). Planification de situations d'apprentissage. Cadre de référence. Fascicule L. Québec.

Ministère de l'Éducation (1988). La résolution de problèmes. Orientations générales. Fascicule K. Québec.

Noël, L.-M., \& Mura, R. (1999). Images des mathématiques chez les futurs maîtres. Revue canadienne de l'éducation, 24(3), 298-312. 
Ross, W. (1987). Teachers' perspective development: A study of preservice social studies teachers. Theory and Research in Social Education, 15, 225-243.

Schram, P., Wilcox, S.K., Lanier, P., \& Lappan, G. (1988). Changing mathematical conceptions of preservice teachers: A content and pedagogical intervention. Washington DC: National Center on Teacher Education Learning.

Steele, D.F. (1994). Helping preservice teachiers confront their conceptions about mathematics and mathematics teaching and learning. Florida: ERIC no. ED 390848.

Strauss, A.L., \& Corbin, J. (1990). Basics qualitative research. Grounded theory procedures and techniques. London: Sage.

Thompson, A.G. (1984). The relationship of teachers conceptions of mathematics and mathematics teaching to instructional practice. Educational Studies in Mathematics, 15(2), 105-127.

Underhill, R.G. (1988). Mathematics teachers' beliefs: Review and reflection. Focus on Learning Problems in Mathematics, 10(3), 43-59.

Vacc Nesbitt, N., \& Bright G.W. (1999) Elementary preservice teachers' changing beliefs and instructional use of children's mathematical thinking. Journal for Research in Mathematics Education, 30(1), 89-110.

Vergnaud, G. (1991). L'enfant, la mathématique et la réalité (4e éd.). Berne: Peter Lang.

Westerman, D. (1991). Expert and novice teacher decision-making. Journal of Teacher Education, 42(4), 292-305.

Wilcox, S.K., Lanier, P., Schram, P., \& Lappan, G. (1992). Influencing beginning teachers' practice in mathemaics education: Confronting constraints of knowledge, beliefs, and context. Washington, DC: National Center on Teacher Education Learning.

Zazkis, R., \& Campbell, S. (1994). Divisibility and multiplicative Structure of natural numbers: Preservice teachers' understanding. Vancouver, BC: ERIC no. ED 370932.

Wideen, M., Mayer-Smith, J., \& Moon, B. (1998). A critical analysis of the research on learning to teach: Making the case for an ecological perspective on inquiry. Review of Educational Research, 68(2), 130-178. 